\title{
Maximum angle of stability of a wet granular pile
}

\author{
SARAH NOWAK ${ }^{1}$, AZADEH SAMADANI ${ }^{1,2}$ AND ARSHAD KUDROLL2* \\ ${ }^{1}$ Department of Physics, Massachusetts Institute of Technology, Cambridge, Massachusetts 02139, USA \\ ${ }^{2}$ Department of Physics, Clark University, Worcester, Massachusetts 01610, USA \\ *e-mail: akudrolli@clarku.edu
}

A nyone who has built a sandcastle recognizes that adding liquid to the sand grains increases the overall stability. However, measurements of the stability in wet granular materials often conflict with theory and with each other ${ }^{1-7}$. The friction-based Mohr-Coulomb model ${ }^{3,8}$ distinguishes between granular friction and interparticle friction, but uses the former without providing a physical mechanism. A frictionless model for the geometric stability of dry particles on the surface of a pile $^{2}$ is in excellent agreement with experiment. However, the same model applied to wet grains overestimates the stability and predicts no dependence on system size. Here we take a frictionless liquid-bridge model and perform a stability analysis within the pile. We reproduce our experimentally observed dependence of the stability angle on system size, particle size and surface tension. Furthermore, we account for past discrepancies in experimental reports by showing that sidewalls can significantly increase the stability of granular material.

The experimental apparatus consisted of a clear plexiglass drum that was rotated about a horizontal axis. We primarily used a drum with a diameter $D$ of $28.5 \mathrm{~cm}$ and a width $W$ that could be varied from 0 to $14.5 \mathrm{~cm}$. A drum with $D=12.5 \mathrm{~cm}$ and $W=11.5 \mathrm{~cm}$ was also used, to vary system size. The rotation rate $\omega$ was varied from $9.0 \times 10^{-4}$ to $5.6 \times 10^{-1}$ r.p.m. Soda-lime glass spheres with density $\rho$ of $2.4 \mathrm{~g} \mathrm{~cm}^{-3}$, radii $r=0.25,0.3,0.5$ and $1.5 \mathrm{~mm}$ and size dispersity within $0.1 \mathrm{~mm}$ were used. The drum was $40 \%$ filled with grains premixed with a small amount of liquid. We report the amount of liquid added in terms of the volume fraction, $V_{\mathrm{f}}$, which is defined as the volume of the liquid divided by the volume occupied by the grains alone. The effect of surface tension of the liquid $\Gamma$ was tested by using silicone oil and water, which have $\Gamma=20 \pm 1$ and $70 \pm 1$ dyn $\mathrm{cm}^{-1}$, respectively. Silicone oil with viscosity $v$ ranging from 5 to $1,000 \mathrm{cS}$ was used to study its impact on the measured inclination angles.

The drum was back lit so that light could pass through a thin layer of grains that tended to accumulate on the sides of the drum, but was unable to pass through the bulk of the pile. Images were acquired with a digital camera of mega-pixel resolution at a rate of three frames per second and these were used to determine the surface slope with automated code. At this rate, the slope of the pile changed by no more than $0.2^{\circ}$ between frames. This error in measurement is not significant given that the slope of the pile at the moment of avalanche was distributed over about two degrees in a given run. At least 30 events were recorded to measure the mean values. The error bars in Figs 2 and 4 correspond to the r.m.s. of the mean angles measured over five experimental runs, each consisting of at least 30 avalanche events.

A stick-slip avalanche regime or a continuous avalanche regime is observed depending on the rotation rate of the drum and the liquid viscosity (see Fig. 1). In the stick-slip regime, the slope of the pile increases linearly with time until the heap reaches the maximum angle $\theta_{\mathrm{m}}$. At this point, the grains are observed to avalanche and the pile's angle decreases to the angle of repose, $\theta_{\mathrm{r}}$, when avalanching stops. In the continuous avalanche regime, the grains are observed to flow continuously and the slope of the pile is approximately constant over time. By changing $\omega$ and $v$, one can change from the stick-slip to the continuous avalanche regime. $\theta_{\mathrm{m}}$ in the stick-slip regime is observed to be independent of $\omega$ and $v$ within the fluctuations in the data and thus is rate independent. On the other hand, $\theta_{\mathrm{r}}$ and the surface angle in the continuous avalanche depend on the viscosity, consistent with other observations ${ }^{6}$. The overall behaviour as a function of $\omega$ is similar to systematic investigations with a rotated $\operatorname{drum}^{7}$, but the magnitudes are significantly lower.

We note that empirically, as a function of $V_{\mathrm{f}}, \theta_{\mathrm{m}}$ increases sharply, and saturates when $V_{\mathrm{f}}$ is of the order of $1 \times 10^{3} \theta_{\mathrm{m}}$ (see Fig. 2a). The dependence of the angles on $V_{\mathrm{f}}$ is again qualitatively consistent with other studies ${ }^{6,7}$, but the overall increase and saturation is significantly lower. At very small $V_{\mathrm{f}}, \theta_{\mathrm{m}}$ can be sensitive to $V_{\mathrm{f}}$ because of the inherent roughness of the grains and its impact on the shape of the liquid bridge ${ }^{1}$. We performed our experiments in the saturation regime, so that such effects were eliminated, but we also chose a small enough $V_{\mathrm{f}}$ so that the liquid did not drain to the bottom because of gravity, which would cause spatial inhomogeneity.

We determined experimentally that higher angles can be attributed to significant wall effects when narrow widths are used, as in most other investigations. For example, the angles reported in ref. 7 are $15-20^{\circ}$ higher than those we report for similar $r$ and $\Gamma$, but the apparatus was $3.2 \mathrm{~cm}$ wide. The observed dependence on $W$ is plotted in Fig. $2 \mathrm{~b}$ and is described by an exponential fit. The 

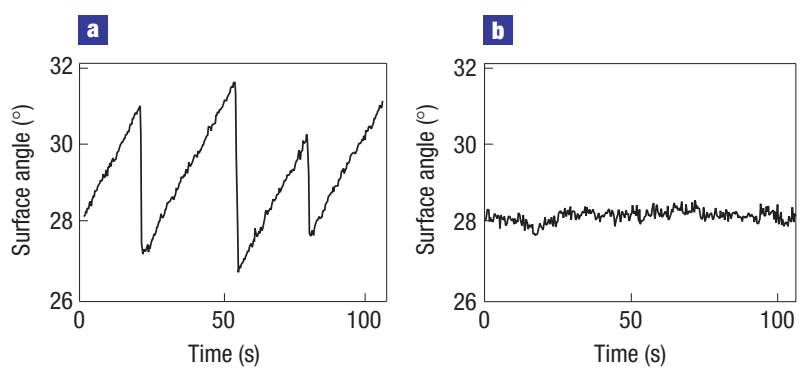

Figure 1 Avalanching regimes. Glass spheres $(r=0.5 \mathrm{~mm})$ mixed with silicone oil in a rotating drum $(W=14.5 \mathrm{~cm})$. a, Stick-slip flow is observed for $\omega=0.028$ r.p.m. and viscosity $v=5 \mathrm{cS}$. b. Continuous flow is observed for $\omega=0.28$ r.p.m. and $v=5 \mathrm{cS}$.

effect of side walls on grains that are fully immersed in a liquid has been reported in ref. 9. The data in that case reach the asymptotic value for a $W$ of a few grain diameters because liquid bridges are absent. However, the side walls have an effect over many hundred times the diameter of the particle in the partially saturated case because of the added particle-particle correlations induced by the liquid bridges. In order to simplify the analysis, we examine the data only for $W>11.5 \mathrm{~cm}$ where the side walls are unimportant.

A $\theta_{\mathrm{m}}$ of at least $90^{\circ}$ is predicted in ref. 2 if the ratio of the capillary to gravitational force, which is also called the bond number (Bo; ref. 5), is of order one or greater. Now the capillary force caused by the liquid-bridge bond is given by

$$
F_{\mathrm{c}}=\alpha \pi \Gamma r,
$$

where $\alpha$ is a dimensionless constant that depends on the size and shape of the liquid bridge between the particles, and their separation $^{10,11}$. Although there is some ambiguity in the value of $\alpha$, it is of order 1, and therefore Bo is approximately 5 for glass beads with $r=0.5 \mathrm{~mm}$ mixed with silicone oil at $V_{\mathrm{f}}=0.008$. We do not observe $\theta_{\mathrm{m}}$ to reach $90^{\circ}$; the piles typically avalanche when $\theta$ exceeds $30^{\circ}$ as can be noted from Fig. 1. We believe that the discrepancy arises from the assumption that a wet granular pile fails at the surface, as was also noted in ref. 3 .

Following ref. 2, let us consider the stability of a sphere placed on top of three base spheres that are all in contact with each other (see Fig. 3). In the absence of liquid bridges, the sphere becomes unstable when the gravitational force is outside the triangle formed by the base spheres. By averaging over all possible orientation angles $\phi$ of the base triangle between 0 and $60^{\circ}$, in ref. 2 it was shown that the average inclination $\theta_{\mathrm{d}}$ (the geometric or dry angle of stability) is $23.8^{\circ}$. At this angle, approximately half of the triangular bases at the surface can support a sphere and thus the pile will be stable. Measurements with dry glass, plastic or steel spheres in a rotated drum all show $\theta_{\mathrm{m}}$ that are within a few degrees of their prediction, and thus it seems that the complications associated with random packing and friction between particles need not be taken into account to obtain reasonable results.

Let us again consider a sphere resting on three base spheres (as in Fig. 3b) that is located at a distance $x$ and angle $\theta$ from the bottom of the inclined surface. For $\theta>\theta_{\mathrm{d}}$, the sphere will be gravitationally unstable. Now we assume that, on average, it is the component of the liquid-bridge bond $a b$ directed up the inclined plane tilted at $\theta$ that is responsible for offsetting the unbalanced gravitation component down the plane. (We neglect the contributions of the bonds $a c$ and $a d$ because they are mostly directed towards the axis around which the top sphere rotates when it becomes unstable.)
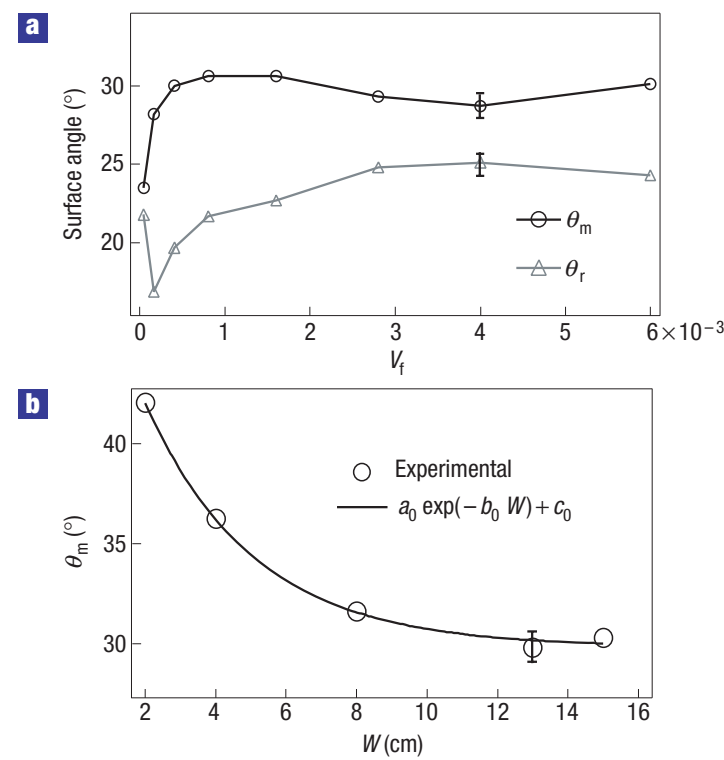

Figure 2 Impact of liquid volume fraction and drum width on stability.

a, Measured surface angles as a function of $V_{\mathrm{f}}$ of silicone oil $(v=5 \mathrm{cS}, r=0.5 \mathrm{~mm}$, $\omega=0.028$ r.p.m.). b, $\theta_{\mathrm{m}}$ is observed to decay exponentially as $W$ is increased. An error bar is indicated on one of the points $\left(V_{\mathrm{f}}=8 \times 10^{-3}\right.$, fitting parameters $\left.a_{0}=23.3^{\circ}, b_{0}=0.33 \mathrm{~cm}^{-1}, c_{0}=30^{\circ}\right)$.

Because $a b c d$ forms a tetrahedron, the projection of the force $F_{\mathrm{c}}$ up the plane of the base triangle because of the liquid bridge between $a b$ can be easily determined. The average component of the liquid bond force corresponding to the average orientation angle $\phi$ of the base triangle is given by $F_{\mathrm{c}} / \sqrt{24} \tan \theta_{\mathrm{d}}$, which is approximately $0.46 F_{c}$. Therefore, the shear stress that can be supported by the liquid bridges along the $\theta$ plane is given by the calculated force per sphere multiplied by the average number of spheres per unit area. Now, the number of particles per unit volume is $3 f_{\mathrm{p}} / 4 \pi r^{3}$, where $f_{\mathrm{p}}$ is the packing fraction of the grains, which is approximately 0.64 for spherical grains. Therefore, the number of particles per unit area is equal to the number of particles per unit volume raised to the $2 / 3$ power. We then have the shear stress

$$
\frac{F_{\mathrm{c}}}{\sqrt{24} \tan \theta_{\mathrm{d}}}\left(\frac{3 f_{\mathrm{p}}}{4 \pi r^{3}}\right)^{2 / 3}
$$

where $F_{\mathrm{c}}$ is given by equation $(1)$.

Now let us calculate the shear stress owing to the unbalanced weight of a thin vertical volume element above a plane tilted at $\theta$ and located at a distance $x$ from the bottom of the surface, as shown in Fig. 3a. We can write the unbalanced weight as

$$
M g \sin \left(\theta-\theta_{\mathrm{d}}\right),
$$

where $M$ is the mass of the volume element that has a length $\mathrm{d} x$, width $W$ and a height $x \sin \left(\theta_{\mathrm{m}}-\theta\right) / \cos \theta_{\mathrm{d}}$, and $g$ is the acceleration due to gravity. We take $\left(\theta-\theta_{\mathrm{d}}\right)$ in equation (2) because the pile is geometrically stable up to $\theta_{\mathrm{d}}$. Thus we find that the shear stress on the $\theta$ plane from the weight of the volume element is

$$
x \rho g f_{\mathrm{p}} \sin \left(\theta_{\mathrm{m}}-\theta\right) \sin \left(\theta-\theta_{\mathrm{d}}\right) / \cos \theta_{\mathrm{d}} .
$$

To simplify further discussion, we take the small-angle approximation, and obtain the unbalanced stress to be

$$
x \rho g f_{\mathrm{p}}\left(\theta_{\mathrm{m}}-\theta\right)\left(\theta-\theta_{\mathrm{d}}\right) / \cos \theta_{\mathrm{d}} .
$$




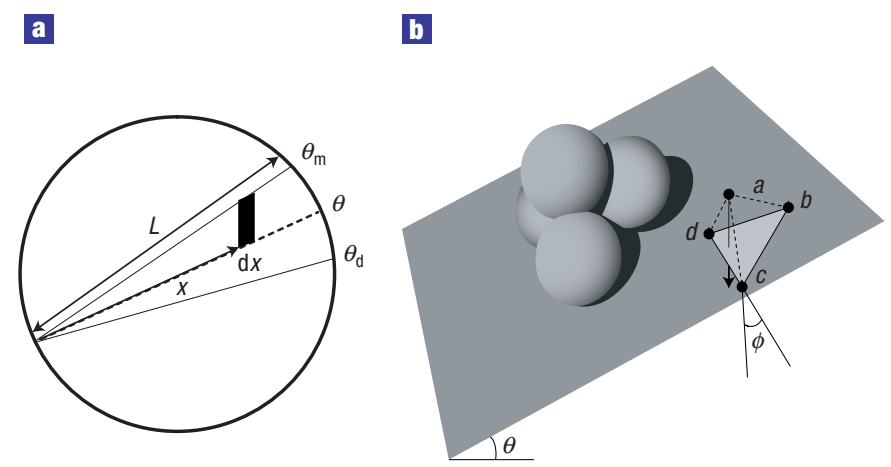

Figure 3 The liquid-bridge model. a, A schematic of the system. b, The geometrical arrangement of one sphere resting on top of three spheres in contact, as used in the stability analysis. Liquid bridges located at the points of contact introduce forces along the segments joining the vertices abcd of the tetrahedron at which the spheres are centred.

Now for each $x$ it can be shown that this shear stress is maximum for $\theta=\left(\theta_{\mathrm{m}}+\theta_{\mathrm{d}}\right) / 2$. This is simply because, as one goes above $\theta_{\mathrm{d}}$, on the one hand there is less material above, but on the other the volume element is tilted further from equilibrium.

It is not clear whether the pile will fail when the shear stress from the weight first exceeds the stress provided by the liquid bridges, or when the average shear stress owing to the weight of the pile exceeds the stress provided by the liquid bridges. In reality, the correct physical situation is most probably a combination of these two. Therefore, we set $x=\beta L$ in equation (4) when balancing the stresses, where $\beta$ is a dimensionless constant between 0.5 and 1 , and $L$ is the length of the pile's surface. If adjacent volume elements cannot offset any stress by a particular volume element then, $\beta=1$, and if the stress is evenly distributed along the slip plane then $\beta=0.5$. Given the variance seen in the stick-slip events (see Fig. 1a), and the heterogeneity of the pile at the granular level, $\beta$ may vary from event to event.

Combining these facts and balancing the two stress components, we obtain the condition for equilibrium,

$$
\left(\frac{\theta_{\mathrm{m}}-\theta_{\mathrm{d}}}{2}\right)^{2}=\left(\frac{9 \pi}{16 f_{\mathrm{p}}}\right)^{1 / 3}\left(\frac{\alpha \cos \theta_{\mathrm{d}} \Gamma}{\sqrt{24} \tan \theta_{\mathrm{d}} \rho g r \beta L}\right),
$$

and, setting $\alpha_{0}=\alpha / \beta$, we obtain

$$
\theta_{\mathrm{m}}-\theta_{\mathrm{d}}=\sqrt{\left(\frac{9 \pi}{2 f_{\mathrm{p}}}\right)^{1 / 3}\left(\frac{\alpha_{0} \cos \theta_{\mathrm{d}} \Gamma}{\sqrt{6} \tan \theta_{\mathrm{d}} \rho g r L}\right)} .
$$

Figure 4a shows a comparison of the measured $\theta_{\mathrm{m}}$ as a function of particle size $r$ and the fit to equation (5) after conversion of the units to degrees. The data are well described by using $\alpha_{0}=1.2 \pm 0.05$. To further test the model, we measured $\theta_{\mathrm{m}}$ in two different-sized systems $(L=27$ and $12.5 \mathrm{~cm})$ with various combinations of $\Gamma$ and $r$. Figure $4 \mathrm{~b}$ shows a plot of the experimentally measured $\theta_{\mathrm{m}}$ above $\theta_{\mathrm{d}}$ compared with equation (5), with $\alpha_{0}=1.2$. Excellent overall agreement is observed.

We note that the small-angle approximation used to simplify equation (3) is reasonable over the angles tested. The analysis can be extended to higher angles without using this approximation, but $\theta_{\mathrm{m}}$ has to be obtained by a numerical solution of the corresponding equation. In developing our analysis, we have ignored the contribution of friction that will also help stabilize the wet pile. However, just as in the dry case, we may expect the friction contribution to be small as a grain rolls after being dislodged from
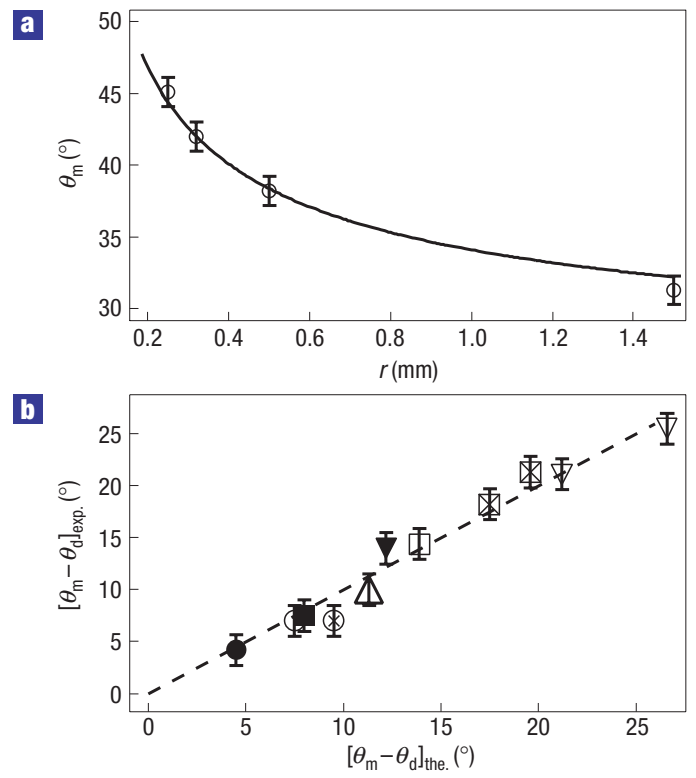

Figure 4 Comparison of the measured data with the liquid-bridge model. a, The maximum angle of stability for glass spheres mixed with water $(L=27 \mathrm{~cm})$. The solid line shows a fit to equation (5) with $\alpha_{0}=1.2$. b. The experimentally measured $\theta-\theta_{\mathrm{d}}$ are plotted against the theoretical values calculated from equation (5). Key: silicone oil in the large drum (circle), water in the large drum (square), silicone oil in the small drum (triangle up), water in the small drum (triangle down). Filled shapes, open shapes and shapes with $\mathrm{a} \times$ sign correspond to radii $r=1.5,0.5$ and $0.3 \mathrm{~mm}$, respectively.

the triangular base. A more detailed knowledge of how the failure develops may clarify this issue.

Our analysis captures the observed stability dependence on grain size, system size and surface tension. It would be of great interest to consider how well this analysis extends to piles composed of non-spherical and multisized grains as in natural sand.

Received 14 April 2005; accepted 2 August 2005; published 29 September 2005.

References

1. Hornbaker, D., Albert, R., Albert, I., Barabási, A. L. \& Schiffer, P. What keeps sandcastles standing? Nature 387, 765 (1997).

2. Albert, R., Albert, I., Hornbaker, D., Schiffer, P. \& Barabási, A. L. Maximum angle of stability in wet and dry spherical media. Phys. Rev. E 56, 6271-6274 (1997).

3. Halsey, T. C. \& Levine, A. J. How sandcastles fall. Phys. Rev. Lett. 80, 3141-3144 (1998).

4. Fraysse, N., Thome, H. \& Petit, L. Humidity effects on the stability of a sandpile. Eur. Phys. J. B 11, 615-619 (1999).

5. Nase, S. T., Vargas, W. L., Abatan, A. A. \& McCarthy, J. J. Discrete characterization tools for cohesive granular materials. Powder Technol. 116, 214-223 (2001).

6. Samadani, A. \& Kudrolli, A. Angle of repose and segregation in cohesive granular matter. Phys. Rev. E 64, 050301 (2001)

7. Tegzes, P., Vicsek, T. \& Schiffer, P. Avalanche dynamics in wet granular materials. Phys. Rev. Lett. 89, 094301 (2002).

8. Nedderman, R. M. Statics and Kinematics of Granular Materials (Cambridge Univ. Press, Cambridge, England, 1992).

9. du Pont, S. C., Gondret, P., Perrin, B. \& Rabaud, M. Wall effects on granular heap stability. Europhys. Lett. 61, 492-498 (2003).

10. Lian, G., Thornton, C. \& Adams, M. J. A theoretical study of the liquid bridge forces between two rigid spherical bodies. J. Colloid Interface Sci. 161, 138-147 (1993).

11. Groger, T., Tuzun, U. \& Heyes, D. M. Modeling and measuring of cohesion in wet granular materials. Powder Technol. 133, 203-215 (2003).

\section{Acknowledgements}

We thank J. Norton and N. Israeloff for their help with the apparatus, and J. Bico for feedback on the manuscript. The work was supported by the National Science Foundation Grant No. DMR-9983659, and the GLUE program of the Department of Energy.

Correspondence and requests for materials should be addressed to A.K.

\section{Competing financial interests}

The authors declare that they have no competing financial interests.

Reprints and permission information is available online at http://npg.nature.com/reprintsandpermissions/ 